\title{
Review Article \\ Hybrid Electronic Tongues Applied to the Quality Control of Wines
}

\author{
Manuel Gutiérrez-Capitán, ${ }^{1}$ Fina Capdevila, ${ }^{2}$ \\ Jordi Vila-Planas, ${ }^{1}$ Carme Domingo, ${ }^{2}$ Stephanus Büttgenbach, ${ }^{3}$ Andreu Llobera, ${ }^{1}$ \\ Anna Puig-Pujol, ${ }^{2}$ and Cecilia Jiménez-Jorquera ${ }^{1}$ \\ ${ }^{1}$ Instituto de Microelectrónica de Barcelona (IMB-CNM), CSIC, Campus UAB, 08193 Bellaterra, Spain \\ ${ }^{2}$ Institut de Recerca i Tecnologia Agroalimentàries-Institut Català de la Vinya i el Vi (IRTA-INCAVI), Plaça Àgora 2-3, \\ 08720 Vilafranca del Penedès, Spain \\ ${ }^{3}$ Institut für Mikrotechnik, Technische Universität Braunschweig, Alte Salzdahlumer Straße 203, 38124 Braunschweig, Germany \\ Correspondence should be addressed to Manuel Gutiérrez-Capitán; manuel.gutierrez@csic.es
}

Received 10 April 2014; Revised 29 July 2014; Accepted 29 July 2014; Published 10 September 2014

Academic Editor: Constantin Apetrei

Copyright (C) 2014 Manuel Gutiérrez-Capitán et al. This is an open access article distributed under the Creative Commons Attribution License, which permits unrestricted use, distribution, and reproduction in any medium, provided the original work is properly cited.

\begin{abstract}
The legislation of food industry is becoming increasingly strict with regard to the quality of food products. Therefore, the market is demanding for automatic systems of analysis that allow fast and accurate monitoring of the evolution of quality parameters in agrofood products or permit obtaining information to optimize production processes. In this context, sensors and more specifically microsensors play an important role since they allow fast and reproducible measurement of a large number of quality parameters with good reliability and can be implemented in portable systems. This paper presents a review of the results obtained with an electronic tongue based on different kinds of microsensors applied to wine analysis by the team of IMB-CNM. This multisensor system allows on one hand classifying the wine according to its features like grape variety, geographic origin, year, and organoleptic characteristics and on the other hand quantifying some parameters of interest in quality control, such as alcoholic degree, $\mathrm{pH}$, ions, total acidity, glycerol, and color.
\end{abstract}

\section{Introduction}

Quality control in agrofood processes is a priority worldwide. Specifically, measurement of some parameters in wine or alcoholic beverages at various stages of production allows taking decisions which affect the final quality of the product. There are commercially available devices that allow quantitative measurement of most parameters related to the quality of wines, such as alcoholic degree, $\mathrm{pH}$, or color. However, these devices require laboratory facilities, they are not portable, and often the cost per analysis is high. Therefore, it should be appealing to develop multiparametric systems that can be portable and of low cost, require low-power consumption, and are able to carry out in situ measurements. Solid-state microsensors meet many of the requirements for these types of systems because of their small size, mass production, fast response, and low power consumption. The combination of these microsensors with chemometric tools for signal processing provides multiparametric analytical systems or hybrid electronic tongues with multiple and attractive applications to food analysis.

An electronic tongue (ET) [1] consists of a set of sensors with low selectivity and advanced mathematical methods based on pattern recognition and multivariate data analysis, such as artificial neural networks (ANN), principal component analysis (PCA), soft independent modeling class analogy (SIMCA), and partial least squares (PLS). These ETs can analyze samples from a qualitative point of view, by means of classification according to the organoleptic characteristics, presence of adulterants or compounds of different nature, type of production, and so forth. Applied to quantitative analysis, the ETs can improve the quality of the 


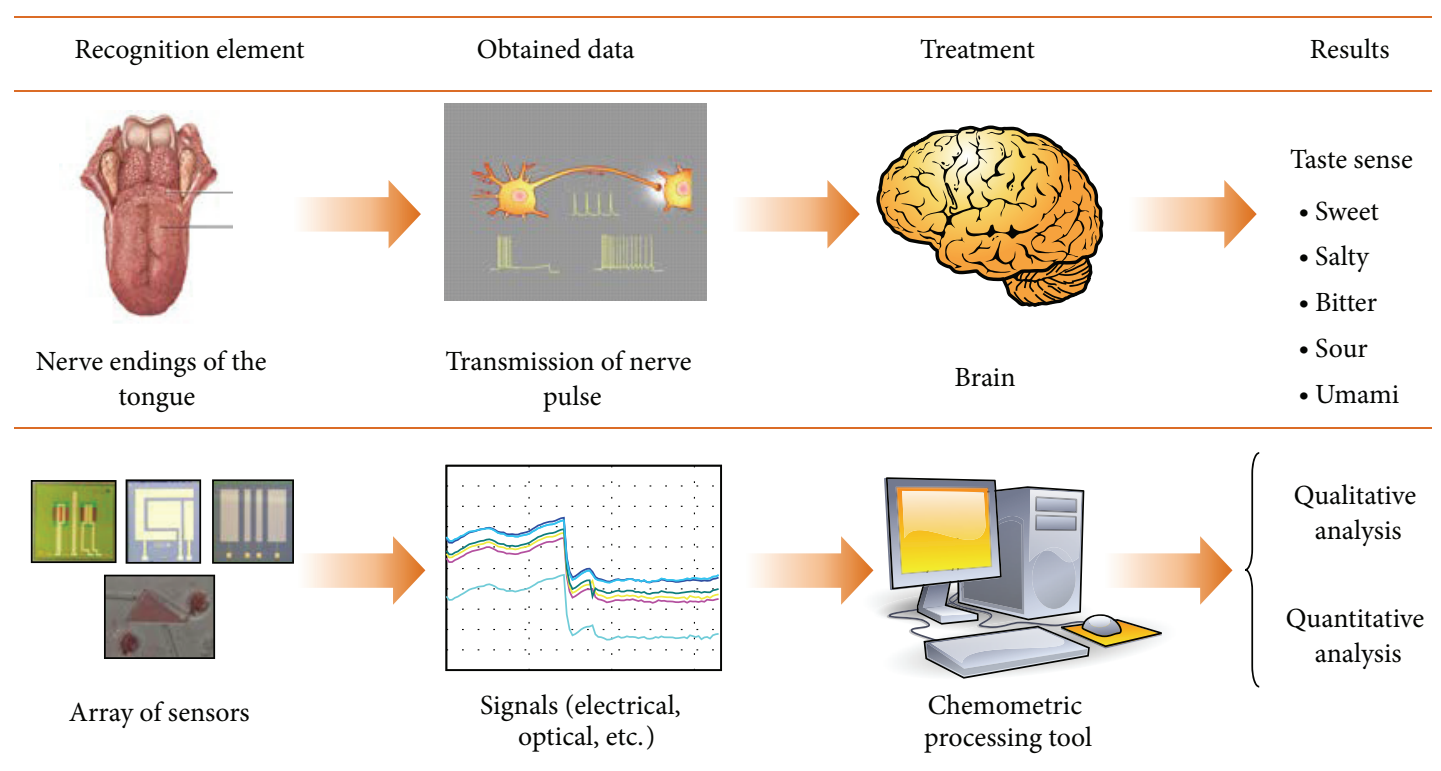

FIGURE 1: Analogy between the electronic tongue and the gustatory organ system.

results, for example, the sensitivity of the sensors. Because of the large number of signals, the system can minimize some limitations of the analysis such as interferences, matrix effects, and detection limits.

The first application of multisensor systems for food classification according to the organoleptic characteristics led to the beginning of the study of these systems under the name of Taste Sensor [2]. Lately, these systems were applied to measure parameters different than organoleptic descriptors and other types of samples and they were renamed electronic tongues [3]. Figure 1 shows the analogy between the ET and the gustatory organ system. As can be seen, the system is formed by an array of sensors, which emulate the nerve endings of the tongue. The signals obtained from these sensors are processed by a chemometric tool in the same way as the brain treats the nerve pulses. The results of the brain treatment are the five basic tastes and the synergies between them, while the results of the chemometric processing are either qualitative or quantitative analysis.

It should be noted that, due to the multidisciplinarity required for these developments, the research groups working on electronic tongues are limited to a few and the most reported applications are in wine [4]. ET systems for classifying grape varieties [5, 6], adulteration [7], or even the time of aging [8] are described. One can also find in the literature ETs applied to quantify parameters of interest for the wine industry [9] or to correlate sensory descriptors of expert panels [10]. However, it should be stressed that the large majority of these systems are optimized for the analysis of monovarietal wine samples. It is well known that the identification and even quantification of different grape varieties in wine blends would be of great interest for the producers [11]. This aspect has also been addressed by our research group.
The use of hybrid electronic tongues, which use sensors of different nature (potentiometry, amperometry, conductance, spectrophotometry, and gas sensing), was described in the late nineties as a powerful tool for improving the results of wine analysis with sensors [12, 13]. Since then, only four hybrid systems applied to wine have been reported, including these incorporating optical measurements [14-17].

Regarding commercial electronic tongues, there are two in the market. One is the Astree, manufactured by AlphaMOS [18], whose sensors are ion-selective field effect transistors (ISFETs) fabricated in the IMB-CNM. The Astree is used by the pharmaceutical industry primarily to study drug formulations from the standpoint of taste. However, its incursion into the market of food quality control is not yet consolidated. The second ET is the result of the research led by Professor Toko and it is manufactured in Japan. The device is called Taste Sensor and it is sold through the company Intelligent Sensor Technology (INSENT) [19]. This equipment is applied to food analysis.

The Chemical Transducers Group of the IMB-CNM has been working in the last decade on the development of ETs for different applications mainly in wines and in grape juice. The objective is to obtain feasible analytical systems for quality control of wines that could be used in the production line as well as in the final product. These ETs contain electrochemical microsensors fabricated with microelectronic technology, optical sensors fabricated with polymer technology, and data processing tools (PCA, PLS, and SIMCA). The results demonstrate that these systems can differentiate wines according to grape variety, vintage year, and geographical origin. They are also able to quantify chemical parameters in wines such as $\mathrm{pH}$, ions, alcohol, and acidity, with an error below 10\% compared to standard methods of analysis. 
TABLE 1: Composition of the reference solution for ISFETs measurements.

\begin{tabular}{lc}
\hline Compound & Concentration $(\mathrm{mM})$ \\
\hline $\mathrm{KNO}_{3}$ & 0.12 \\
$\mathrm{KH}_{2} \mathrm{PO}_{4}$ & 5.26 \\
$\mathrm{MgSO}_{4}$ & 4.41 \\
$\mathrm{CaCl}_{2}$ & 2.26 \\
$\mathrm{Malic}$ acid & 19.00 \\
Potassium bitartrate & 33.00 \\
$\mathrm{NaOH}$ & 13.91
\end{tabular}

\section{Materials and Methods}

2.1. Microsensors Used. The used electrochemical sensors were fabricated with silicon technology in the Clean Room of IMB-CNM. These were ISFETs [20], Pt 4-electrode sensors [21], and gold amperometric microelectrodes [22]. The ISFETs allowed measuring $\mathrm{pH}$ and $\mathrm{Na}^{+}, \mathrm{K}^{+}, \mathrm{Ca}^{2+}, \mathrm{Cl}^{-}$, and $\mathrm{NO}_{3}{ }^{-}$ions in solution [23-25]. In order to make the ISFETs selective to these ions, the sensing surface was modified with polymeric membranes. The Pt 4-electrode sensors were used for measuring conductivity and redox potential (ORP) and finally $\mathrm{Au}$ amperometric sensors were used for measuring oxidoreduction currents at several potentials.

The optical sensors were fabricated with soft lithography techniques using the polymer polydimethylsiloxane (PDMS) as the constituent material. The device consists of a hollow prism which can be filled with wine by two fluidic ports. Two biconvex lenses modify the optical path before and after the propagation of light through the prism [26]. The measurement is performed by absorption in the visible rangebetween $200 \mathrm{~nm}$ and $1000 \mathrm{~nm}$-although it is expandable in function of the input light and the optical reader (it could reach NIR values).

2.2. Reagents, Solutions, and Wine Samples. All the reagents used in these studies were of high purity and analytical grade or equivalent. All solutions were prepared with deionized water. Furthermore, for ISFETs measurement, a reference solution containing $12 \%$ of ethanol and an average concentration of the main compounds present in wine was prepared. The components of this solution and the concentrations are shown in Table 1.

Regarding the wine samples, studies were carried out with different sets of red and white wines provided by the Catalan Institute of Vineyard and Wine (INCAVI), which is the official regulatory agency of the Generalitat de Catalunya. The set of white wines consisted of 18 monovarietal (100\% one grape variety) samples based on the following grape varieties: 5 Macabeu, 5 Parellada, 4 Chardonnay, and 4 Xarel.lo. The set of red wines was formed by 12 monovarietal samples based on 4 Merlot, 3 Cabernet Sauvignon, 3 Grenache, and 2 Trepat varieties. Certain chemical and optical parameters in these wines were determined by the INCAVI using standard methods. These methods are dictated by the European Union [27] and are well established by the INCAVI laboratories. The routine protocol consists of one measurement for sample, without repetitions.

Furthermore, a study was performed with 20 mixtures using three red grape varieties: Cabernet Sauvignon, Merlot, and Pinot Noir, in different proportions (15\%, 25\%, 50\%, $75 \%, 85 \%$, and $100 \%$ ). The base wines were from the 2008 vintage, harvested in Penedès (Spain) and collected when the malolactic fermentation was completed. The percentages of each variety were determined using a simplex-lattice experimental design, where the proportions of the different components sum 100\%. For each blend, triplicate samples were prepared resulting in 60 samples.

2.3. Methodology. The wine samples were analyzed without dilution with the different sensors: ISFETs selective to $\mathrm{pH}$ and several ions, $\mathrm{Pt}$ 4-electrode sensors for measuring conductivity and ORP, the amperometric microelectrode for measuring reduction-oxidation reactions, and the hollow prism, that provides color measurement. Each of these sets of sensors has its own instrumentation of measurement and data collection, so that the samples were analyzed sequentially. In the case of the optical system, measurements were carried out by filling the system directly with the wine. In order to obtain the spectra in absorbance units, deionized water was used as reference and it was measured before and after each wine sample. The light was emitted from broadband light source (Ocean Optics HL-2000, Oracle, USA). The readout multimode optical fiber was connected to a spectrometer (Ocean Optics HR4000) with a spectral resolution of $2 \mathrm{~nm}$. For each wine sample, the average of 10 consecutive scans was considered. Therefore, the complete measurement of a wine sample was performed in approximately 15 minutes. No replications of each sample were done following the INCAVI protocol and also to get a rapid analysis and prevent changes of the wine sample.

The characterization of the analytical system was performed by measuring a set of wine samples with known values of the considered parameters. This process is called training and the result is a response model for further study of unknown wines. If the analyzed parameter is qualitative (e.g., grape variety), a multivariate analysis of the sensor data using PCA is performed [28]; therefore data of wine samples are placed in a two-dimensional space that coincides with the directions of maximum variation between them. This distribution method classifies the samples according to the considered parameter. Once the system has been characterized, the signals of an unknown sample are interpolated in the PCA to obtain their properties.

In order to demonstrate the viability of the hybrid ET to discriminate between grape varieties, another chemometric method called SIMCA was used [28]. This method is very useful to predict objectively and statistically whether a sample belongs to a class or group defined a priori. In this case, the response model is based on the similarities between samples of the same group.

To perform a quantitative analysis of a chemical parameter it is also necessary to calibrate the system using a linear treatment like PLS [28]. In this case, the signals from all sensors and the training samples are related to the 


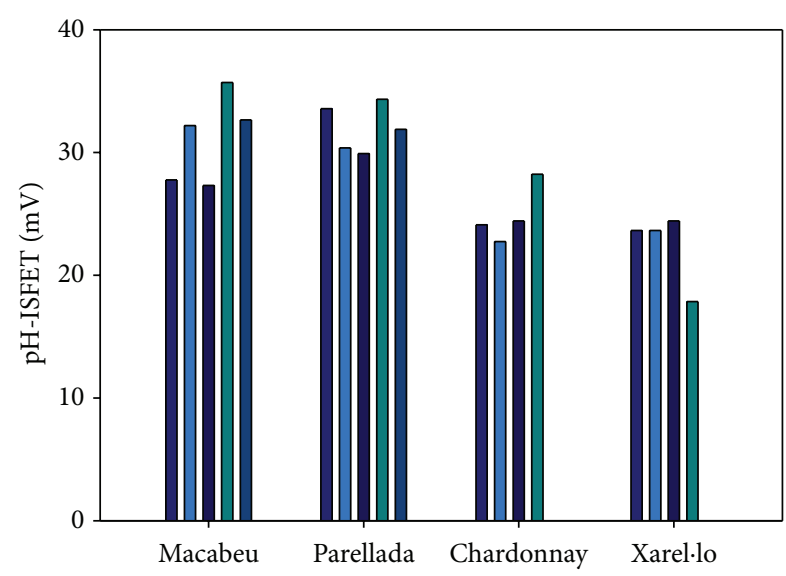

FIGURE 2: pH ISFET signals for a set of white wines with different grape varieties.

values obtained by a reference method of analysis. Then the unknown sample is interpolated in the PLS model to obtain the desired parameter value. The unknown samples are not involved at any point in the calibration. Unlike a uniparametrical calibration, a model for every parameter can be generated using the same sensor signals, without additional experiments.

Finally, the INCAVI provided the results of descriptors of nine white wines. The wines were evaluated by a panel of eight tasters with extensive experience in wine sensory evaluation. A descriptive analysis of each wine was performed in a room set in accordance with ISO 8589 [29]. Sight, smell, and taste descriptors were evaluated by the panelists, assigning a value ranging from 1 (no intensity) to 9 (maximum intensity). The sensory attributes used for this analysis were global, fruity, floral, spice, and vegetal flavours, as well as global taste, acidity, and color quality. Samples were tasted in a randomized order. Wines were presented to the panelists in tasting glasses (NF V09-110 AFNOR, 1995) marked with twodigit random numbers. Tasting was performed at $20-22^{\circ} \mathrm{C}$, and water was provided to rinse the palate between tastings. The final marks of the panel were used as reference data to train the ET using PLS and to evaluate the correlation with the system outputs.

\section{Results and Discussion}

3.1. Election of the Variables. One of the most important steps in a multivariate analysis is the identification of the input variables. The aim is to get the maximum information from the sample, but with the minimum number of variables (sensors) in order to simplify the experimental method and the response model. In the case of the ISFETs, the input data corresponded to the relative signal of each ISFET with respect to the synthetic reference solution. This standard solution was measured before each wine sample in order to prevent the drift and the matrix effects, which may distort the signal. As an example, Figure 2 shows the signals (in $\mathrm{mV}$ ) obtained by the $\mathrm{pH}$-ISFET for a set of 18 white wines. As can be observed, the sensor signal is different for each sample, but there is certain homogeneity for the wines of the same grape variety.

For the conductivity and ORP sensors, the data used in the model were the signals obtained directly from the sensors after 3-minute recording. For the amperometric gold microelectrode, cyclic voltammograms were carried out to obtain the input data for the model. As an example, Figure 3(a) shows the voltammetric signal obtained for four monovarietal white wines: Macabeu, Parellada, Xarel-lo, and Chardonnay. The two redox peaks correspond to the oxidation and reduction of gold at $+1.3 \mathrm{~V}$ and $+0.65 \mathrm{~V}$, respectively. These peaks indirectly provide information of the sample matrix. In addition, two smaller peaks are observed at $+1.0 \mathrm{~V}$ and $-0.35 \mathrm{~V}$ with different current intensities depending on the wine. These peaks can be related to the amount of polyphenols. Therefore, the intensity of these four peaks was used as variables. These values were similar for red wines (see Figure 2 in [17]). These electrochemical variables were the most significant for white wines for the construction of the chemometric models.

Finally, the absorbance spectra of wines were analyzed to determine the optical variables. Figure 3(b) shows the signal obtained for four monovarietal red wines, using deionized water as reference. As can be seen, the spectra are very different between the different grape varieties: Grenache, Trepat, Cabernet Sauvignon, and Merlot. In order to choose the variables for red wines, we applied the EU method that defines the color of wine at absorbance of 420, 520, and $620 \mathrm{~nm}$ [27]. These values were also used for white wines. The value at $920 \mathrm{~nm}$ was also elected for red wines to build the model considering the high variation between samples. It is noteworthy that these optical variables were the most significant for the construction of the red wine models.

A data matrix was constructed with the signals obtained with all these sensors. The differences between white and red wines are too much great that it was necessary to analyze and treat the two sets of samples separately. For all the chemometric methods used, the original values were previously autoscaled (all the variables were centered and set to a standard deviation equal to 1 ) to avoid the fact that the variables have different influence on the model. Then the matrix was introduced into the Unscrambler statistical program (CAMO, Norway) to obtain the processed models.

3.2. Qualitative Analysis of Grape Varieties. The first study was to test if the system was able to classify wines according to the grape variety. The sensor signals were treated using the PCA method. Figure 4(a) shows the representation of the results obtained for the monovarietal white wines and Figure 4(b) shows those for the monovarietal red wines. As can be seen, the system is capable of differentiating between different varieties of grapes with a good capacity for separation. In the model for white wines, it is also observed that the Macabeu wines from different regions of Catalonia are in different groups and distinguished from the other samples [30]. In the case of the red wines, there is a differentiation of the Merlot samples from different vintages (2007 and 2008) [17], which demonstrates the ability of the system to 


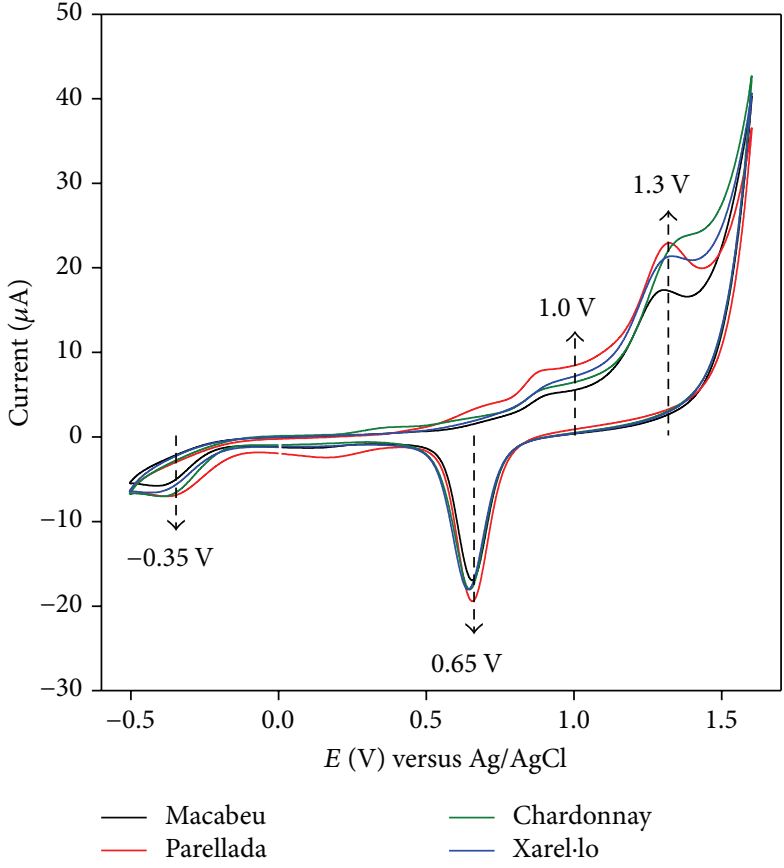

(a)

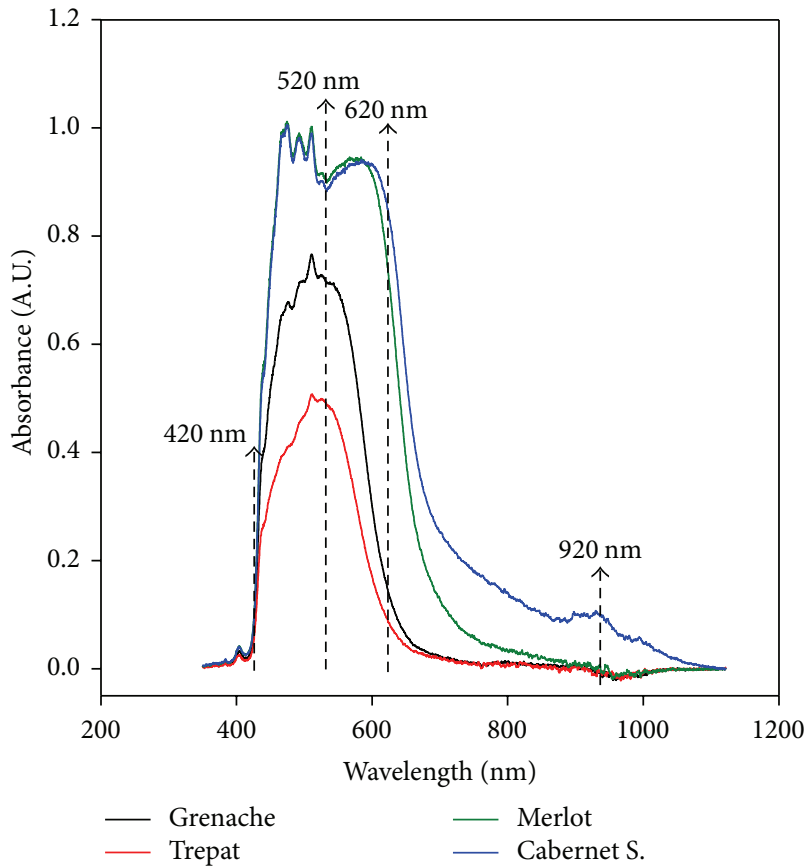

(b)

FIGURE 3: Cyclic voltammograms obtained for a set of white wines (a) and spectra of absorbance obtained for a set of red wines (b). The selected variables are indicated in each case.

distinguish the geographical origin and the year of the wine samples.

This system is also able to differentiate the samples according to the percentage of grape variety in bi- or trivarietal wines [31]. In the study performed with 20 triplicate mixtures of Cabernet Sauvignon, Merlot, and Pinot Noir, the distribution of samples shown in Figure 5 was obtained. As depicted there is a clear distinction between mixtures containing a percentage greater than or equal to $75 \%$ of the main grape variety. Even in the case of Pinot Noir it was possible to distinguish the monovarietal samples (100\%) from those with 85 or $75 \%$. The bi- and trivarietal blends with percentages below 75\% were all situated in the centre of the PCA plot, indicating the feasibility of the model.

For this study with bi- and trivarietal wines, also a SIMCA analysis was performed to check if the system was able to distinguish statistically between the monovarietal wines and the mixtures thereof. One way to visualize the results of a SIMCA analysis is through a Coomans diagram as shown in Figure 6. The $x$-axis represents the distance to the Pinot Noir (100\%) model and the $y$-axis represents the distance to the Merlot (100\%) model. That is, the closer to 0 the $x$ value of a sample is, the more similar to a monovarietal Pinot Noir it is, and the closer to 0 the $y$ value is, the more similar to a monovarietal Merlot it is. Up to a value of 2.9 there is a probability of $90 \%$ that the wine is monovarietal. Therefore, up to a value of 2.9 the system considers all samples as $100 \%$. Therefore, in this particular example, the three $100 \%$ Pinot Noir wines are located within their
TABLE 2: PLS regression data for the comparison of obtained versus expected percentage for the three considered grape varieties for prediction set of mixtures (intervals calculated at the $95 \%$ confidence level).

\begin{tabular}{lccc}
\hline Grape variety & Correlation $\left(r^{2}\right)$ & Slope & Intercept (\%) \\
\hline Pinot Noir & 0.976 & $0.96 \pm 0.08$ & $1.6 \pm 3.7$ \\
Merlot & 0.949 & $0.94 \pm 0.11$ & $3.0 \pm 4.7$ \\
Cabernet Sauvignon & 0.939 & $0.90 \pm 0.12$ & $4.5 \pm 5.7$ \\
\hline
\end{tabular}

own model and well separated from the other mixtures. It is even possible to differentiate the samples with $85 \%$ and $75 \%$ Pinot Noir. In the case of the Merlot model, a few samples with a percentage higher than $75 \%$ of Merlot are confused with the monovarietal ones. It is interesting to note that a great difference in organoleptic characteristics is also reported between Pinot Noir and Merlot.

A PLS regression was performed to evaluate the system's ability to quantify the percentage of each grape variety in these mixtures. Table 2 summarizes the results obtained for the prediction set of samples, those wine mixtures that have not participated in the calibration, for each of the three models: Pinot Noir, Merlot, and Cabernet Sauvignon. The linear regression compares the percentage obtained by the system with the expected percentage for each grape variety. As can be seen, in all cases confidence intervals calculated at $95 \%$, including unity slope and zero intercept, showed no significant differences between the obtained and expected 


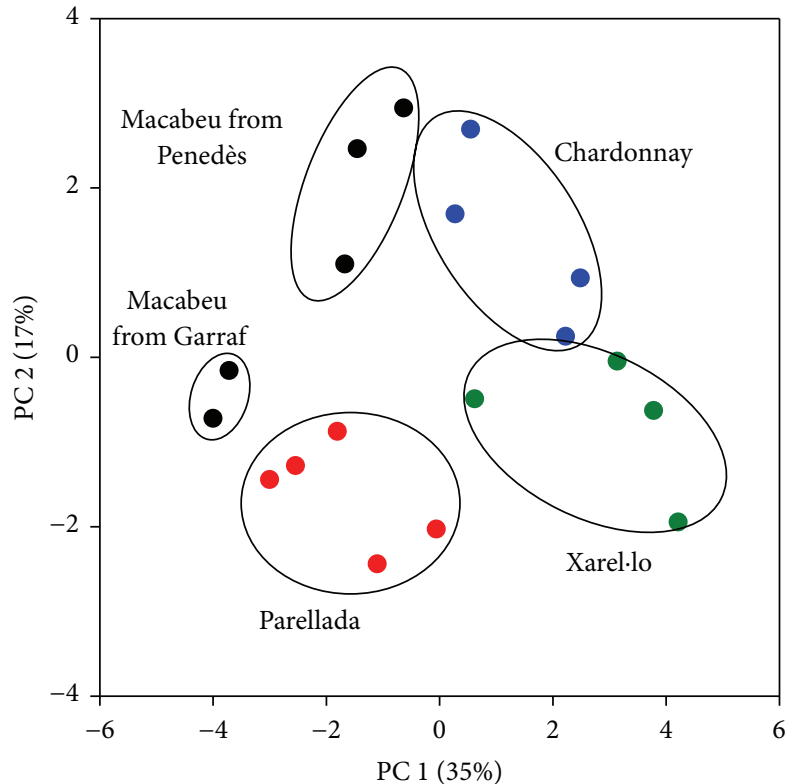

(a)

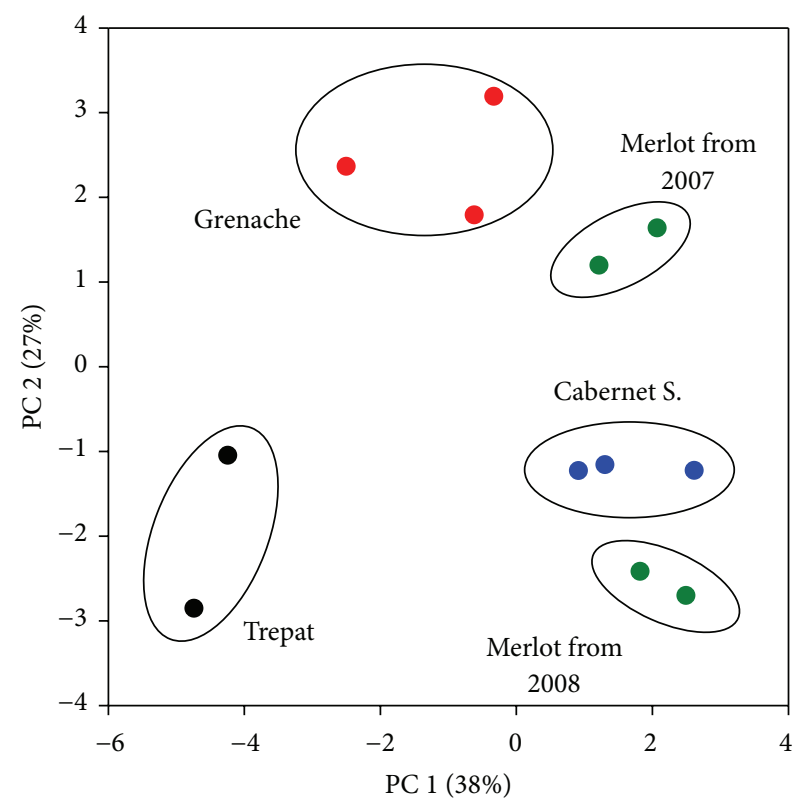

(b)

Figure 4: Representation of the PCA results for a set of white wines (a) and for a set of red wines (b).

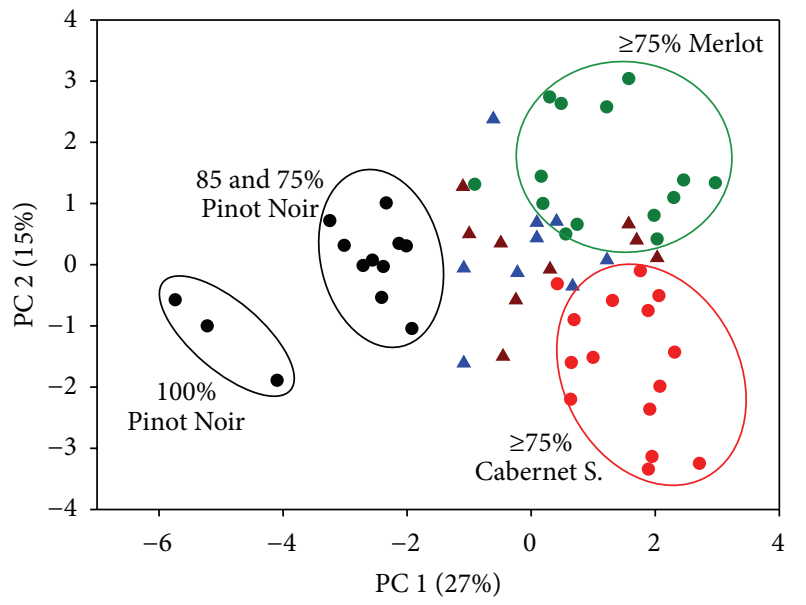

\ Mixtures 50\%: 50\%

^ Trivarietal mixtures

FIGURE 5: Representation of the PCA results for the 20 triplicate mixtures of Cabernet Sauvignon, Merlot, and Pinot Noir.

values. In addition, the correlation coefficients are quite significant.

3.3. Quantitative Analysis. Wine samples were also measured to obtain a quantitative analysis for several parameters of interest in wine quality control $[17,30]$. For that, PLS regression was performed with a set of white and red wines. In Tables 3 and 4 the relative error calculated is shown when comparing the values obtained using the multisensor system and those using standard analytical techniques for the test-set samples. As noted, low errors were obtained for

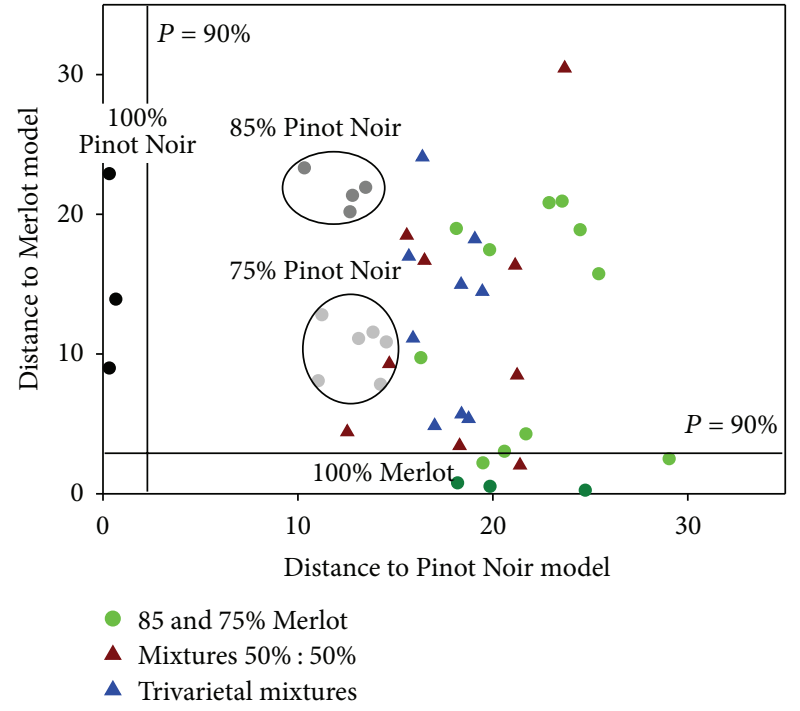

Figure 6: Coomans diagram for the combination of Pinot NoirMerlot models with a probability of $90 \%$.

all parameters $(<7 \%)$, but for particular parameters such as $\mathrm{pH}$ and volumetric alcoholic degree (VAD) this error is even lower. The $\mathrm{pH}$ results are quite obvious because the system contains sensors for measuring $\mathrm{pH}$ (ISFET). However, there are no specific sensors for glycerol, total acidity, and VAD; therefore the results are quite satisfactory.

The use of optical sensors allowed determining wine color parameters, such as intensity of color, tonality, and CIELab L. Table 5 shows the relative errors obtained for a set of white wines. The low errors obtained for the determination 
TABLE 3: Relative errors obtained in the quantification of chemical parameters using the PLS regression for a set of white wines.

\begin{tabular}{|c|c|c|c|c|c|c|}
\hline Sample & VAD & Total acidity & $\mathrm{pH}$ & {$\left[\mathrm{Ca}^{2+}\right]$} & {$\left[\mathrm{Mg}^{2+}\right]$} & Glycerol \\
\hline Macabeu & 2.82 & 3.7 & 0.79 & 0.7 & 4.9 & 6.0 \\
\hline Parellada & 0.07 & 9.5 & 3.55 & 5.1 & 0.1 & 2.0 \\
\hline Chardonnay & 3.32 & 2.7 & 1.16 & 5.7 & 2.2 & 4.3 \\
\hline Xarel.lo & 0.62 & 1.0 & 4.76 & 4.0 & 2.8 & 12.3 \\
\hline Mean error (\%) & 1.71 & 4.2 & 2.56 & 3.9 & 2.5 & 6.2 \\
\hline
\end{tabular}

TABLE 4: Relative errors obtained in the quantification of chemical parameters using the PLS regression for a set of red wines.

\begin{tabular}{|c|c|c|c|c|c|c|}
\hline Sample & VAD & Total acidity & $\mathrm{pH}$ & {$\left[\mathrm{Ca}^{2+}\right]$} & {$\left[\mathrm{K}^{+}\right]$} & Glycerol \\
\hline Trepat & 4.45 & 0.6 & 2.20 & 5.1 & 4.0 & 8.8 \\
\hline Grenache & 4.05 & 7.7 & 1.34 & 6.1 & 6.7 & 2.3 \\
\hline Merlot 2007 & 2.31 & 2.6 & 0.91 & 3.9 & 0.7 & 3.8 \\
\hline Merlot 2008 & 6.52 & 8.0 & 2.03 & 2.4 & 3.2 & 5.6 \\
\hline Cabernet S. & 0.99 & 9.0 & 4.01 & 8.7 & 8.5 & 2.7 \\
\hline Mean error (\%) & 3.66 & 5.6 & 2.10 & 5.2 & 4.6 & 4.6 \\
\hline
\end{tabular}

of CIELab L* parameter and the errors below $5 \%$ for the determination of the others are remarkable.

These quantitative results confirm the capacity of the multisensor system for measuring multiple parameters with acceptable accuracy. It is noteworthy that some of these parameters are obtained by the combination of nonspecific sensors and chemometric methods. This confers a great versatility to the system given that it can measure the desired parameters after a good training of the system.

3.4. Correlation to the Taste Panel. Finally, nine white wines were tested with the electronic tongue to obtain a correlation to the scores of human sensory assessments. Eight sensory parameters were studied by trained panelists and used to construct a PLS model of prediction. The best results were obtained for four descriptors: the global flavour, global taste, acidity, and color, with mean relative errors of $7.2 \%$, $7.4 \%, 4.2 \%$, and $2.4 \%$, respectively. Bars graphs with the outputs of the ET and the taste panel scores for these four parameters are shown in Figure 7. These four parameters are related to global perceptions of sight, smell, and taste. Therefore the nonspecific sensors used, which respond to several chemical compounds and their combinations, are able to extract general characteristics of wine matrix, obtaining a good correlation. For the rest of flavours scored (floral, fruity, vegetal, and spice), the relative errors obtained were above $20 \%$ in general. This could be explained by the fact that these descriptors are related to volatile compounds and very special tastes and the ET is not able to distinguish.

\section{Conclusions}

The results obtained with this hybrid electronic tongue demonstrate that the combination of different kinds of sensors with chemometric tools for signal processing offers a great challenge in the area of food quality control. These systems could be applied to classify food based on the composition, to detect abnormalities in the production process, to set the homogeneity of the raw material and the final product and even to confirm that the parameters are within legal or required limits, and so forth. Those different parameters can be defined previously and the ET is trained (calibrated) accordingly. Therefore this is a highly versatile instrument since it can be adapted to different applications and requirements of the customer.

Regarding the potentiality as analyzers, the ETs can measure a large number of parameters with a limited number of sensors and with high precision and reliability and with high throughput, making the pretreatment of the sample unnecessary. Currently there is nothing similar in the market except colorimetric systems using Fourier transform processing. These systems measure the absorbance of the sample in a wide range of wavelengths, which are then compressed and correlated with the concentration of various parameters. In comparison, the advantages of the ET are a greater versatility due to the different nature of the sensors and a better accuracy for parameters for which selective sensors exist. Besides, an important point is that, with an ET, qualitative and quantitative analyses are obtained using the same experimental data.

The results so far demonstrate that the microsensor technology is suitable for the development of portable equipment and applicable for in situ monitoring. This aspect is important given that currently almost all the analytical methods of the food industry are based on conventional laboratory techniques-distillations, titrations, extractions, and so forth-and on analytical techniques that require complex equipment. 
TABLE 5: Relative errors obtained for the quantification of color parameters using the PLS regression for a set of white wines.

\begin{tabular}{lccc}
\hline Sample & Intensity of color & Tonality & CIELab L* $^{*}$ \\
\hline Macabeu & 4.67 & 1.09 & 0.05 \\
Parellada & 4.56 & 3.66 & Not provided \\
Chardonnay & 0.87 & 2.48 & 0.46 \\
Xarel.lo & 7.29 & 4.23 & 0.10 \\
Mean error (\%) & $\mathbf{4 . 3 5}$ & $\mathbf{2 . 8 6}$ & $\mathbf{0 . 2 0}$ \\
\hline
\end{tabular}

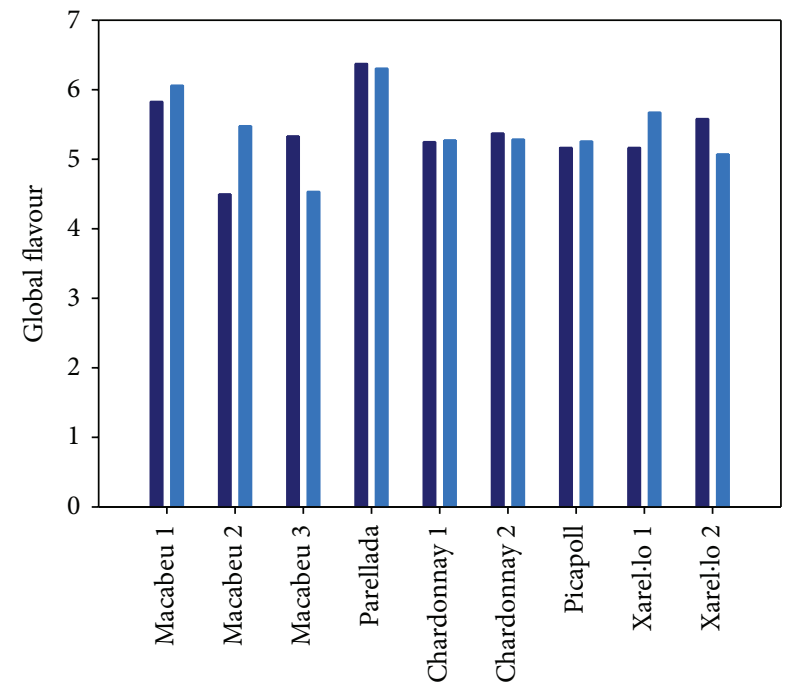

(a)

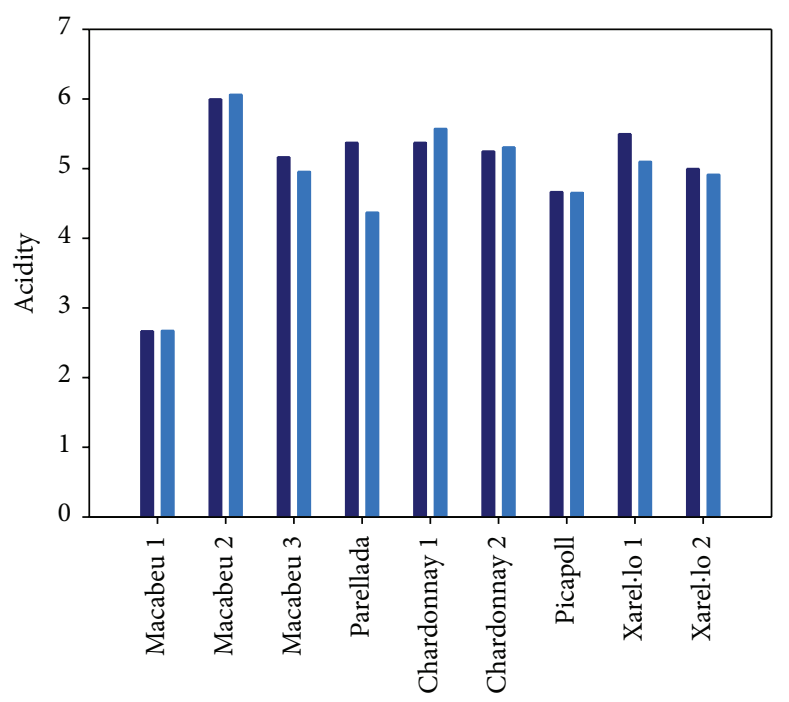

Taste panel score Electronic tongue

(c)

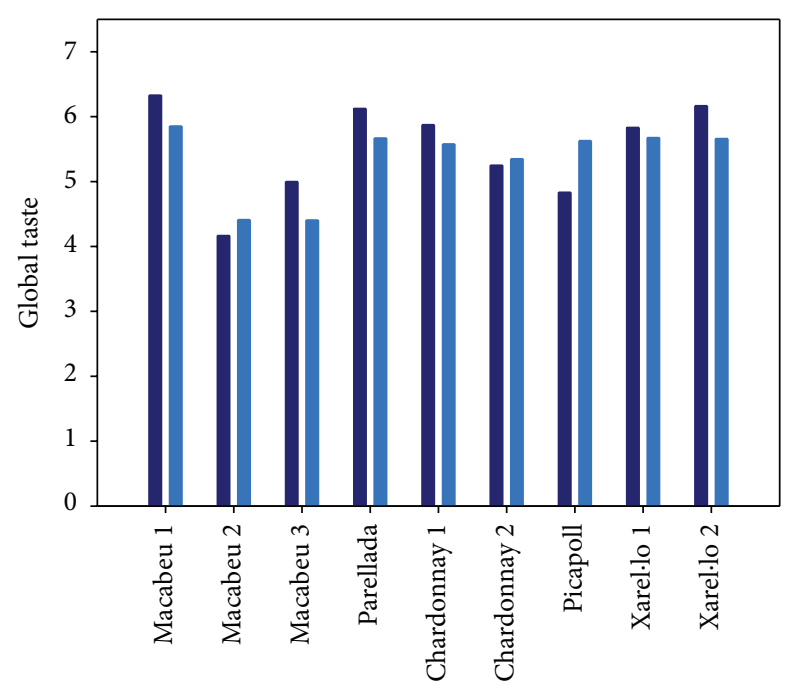

(b)

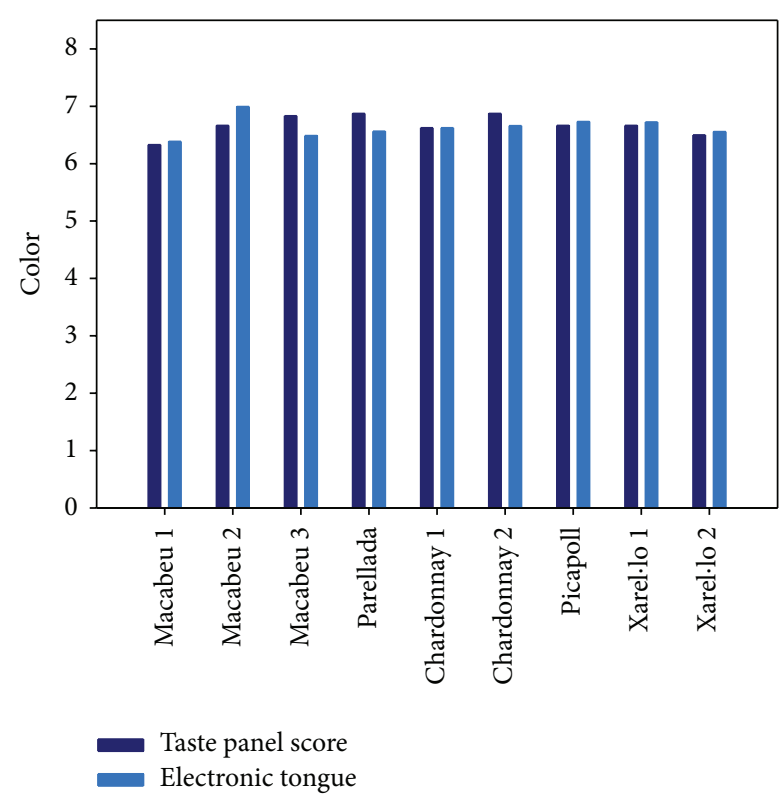

(d)

FIGURE 7: Bars graphs with the correlation results between the taste panel and the ET. 


\section{Conflict of Interests}

The authors declare that there is no conflict of interests regarding the publication of this paper.

\section{Acknowledgments}

The authors acknowledge funding from the Spanish R\&D National Program (MINECO Project TEC2011-29045-C0401/04). The technical assistance of Nidia Santamaría, Alfredo Cadarso, and Héctor Cabezas is highly appreciated.

\section{References}

[1] Y. Vlasov, A. Legin, A. Rudnitskaya, C. Di Natale, and A. D'Amico, "Nonspecific sensor arrays ("electronic tongue") for chemical analysis of liquids (IUPAC Technical Report)," Pure and Applied Chemistry, vol. 77, no. 11, pp. 1965-1983, 2005.

[2] K. Toko, "Taste sensor with global selectivity," Materials Science and Engineering C, vol. 4, no. 2, pp. 69-82, 1996.

[3] M. Holmberg, M. Eriksson, C. Krantz-Rülcker et al., "2nd workshop of the Second Network on Artificial Olfactory Sensing (NOSE II)," Sensors and Actuators B, vol. 101, no. 1-2, pp. 213223, 2004.

[4] J. Zeravik, A. Hlavacek, K. Lacina, and P. Skládal, "State of the art in the field of electronic and bioelectronic tongues-towards the analysis of wines," Electroanalysis, vol. 21, no. 23, pp. 25092520,2009 .

[5] J. M. Gutiérrez, L. Moreno-Barón, M. I. Pividori, S. Alegret, and M. del Valle, "A voltammetric electronic tongue made of modified epoxy-graphite electrodes for the qualitative analysis of wine," Microchimica Acta, vol. 169, no. 3, pp. 261-268, 2010.

[6] W. Novakowski, M. Bertotti, and T. R. L. C. Paixão, "Use of copper and gold electrodes as sensitive elements for fabrication of an electronic tongue: discrimination of wines and whiskies," Microchemical Journal, vol. 99, no. 1, pp. 145-151, 2011.

[7] V. Parra, Á. A. Arrieta, J.-A. Fernández-Escudero, M. L. Rodríguez-Méndez, and J. A. de Saja, "Electronic tongue based on chemically modified electrodes and voltammetry for the detection of adulterations in wines," Sensors and Actuators B: Chemical, vol. 118, no. 1-2, pp. 448-453, 2006.

[8] A. Rudnitskaya, S. M. Rocha, A. Legin, V. Pereira, and J. C. Marques, "Evaluation of the feasibility of the electronic tongue as a rapid analytical tool for wine age prediction and quantification of the organic acids and phenolic compounds. The case-study of Madeira wine," Analytica Chimica Acta, vol. 662, no. 1, pp. 82-89, 2010.

[9] I. Campos, R. Bataller, R. Armero et al., "Monitoring grape ripeness using a voltammetric electronic tongue," Food Research International, vol. 54, no. 2, pp. 1369-1375, 2013.

[10] D. Kirsanov, O. Mednova, V. Vietoris, P. A. Kilmartin, and A. Legin, "Towards reliable estimation of an "electronic tongue" predictive ability from PLS regression models in wine analysis," Talanta, vol. 90, pp. 109-116, 2012.

[11] M. A. Faria, R. Magalhães, M. A. Ferreira, C. P. Meredith, and F. Ferreira Monteiro, "Vitis vinifera must varietal authentication using microsatellite DNA analysis (SSR)," Journal of Agricultural and Food Chemistry, vol. 48, no. 4, pp. 1096-1100, 2000.
[12] S. Baldacci, T. Matsuno, K. Toko, R. Stella, and D. De Rossi, "Discrimination of wine using taste and smell sensors," Sensors and Materials, vol. 10, no. 3, pp. 185-200, 1998.

[13] K. Toko, "A taste sensor," Measurement Science and Technology, vol. 9, no. 12, pp. 1919-1936, 1998.

[14] C. Di Natale, R. Paolesse, A. MacAgnano et al., "Application of a combined artificial olfaction and taste system to the quantification of relevant compounds in red wine," Sensors and Actuators B: Chemical, vol. 69, no. 3, pp. 342-347, 2000.

[15] M. L. Rodríguez-Ménde, A. A. Arrieta, V. Parra, and A. Bernal, "Fusion of three sensory modalities for the multimodal characterization of red wines," Sensors Journal IEEE, vol. 4, no. 3, pp. 348-354, 2004.

[16] S. Buratti, D. Ballabio, S. Benedetti, and M. S. Cosio, "Prediction of Italian red wine sensorial descriptors from electronic nose, electronic tongue and spectrophotometric measurements by means of Genetic Algorithm regression models," Food Chemistry, vol. 100, no. 1, pp. 211-218, 2007.

[17] M. Gutiérrez, A. Llobera, J. Vila-Planas et al., "Hybrid electronic tongue based on optical and electrochemical microsensors for quality control of wine," Analyst, vol. 135, no. 7, pp. 1718-1725, 2010.

[18] http://www.alpha-mos.com/analytical-instruments/astree-electronic-tongue.php.

[19] http://www.insent.co.jp/en/index.html.

[20] C. Jimenez, A. Baldi, N. Abramova, and A. Bratov, "ISFET based sensors: fundamentals and applications in," in Encyclopedia of Sensors, C. A. Grimes, E. C. Dickey, and M. V. Pishko, Eds., pp. 151-196, American Scientific Publishers, 2005.

[21] J. Orozco, A. Baldi, P. L. Martín, A. Bratov, and C. Jiménez, "Monitoring of bentonite pore water with a probe based on solid-state microsensors," Analytica Chimica Acta, vol. 579, no. 1, pp. 95-101, 2006.

[22] R. Olivé-Monllau, J. Orozco, C. Fernández-Sánchez et al., "Flow injection analysis system based on amperometric thinfilm transducers for free chlorine detection in swimming pool waters," Talanta, vol. 77, no. 5, pp. 1739-1744, 2009.

[23] C. Jiménez, I. Marqués, and J. Bartrolí, "Continuous-flow system for on-line water monitoring using back-side contact ISFET-based sensors," Analytical Chemistry, vol. 68, no. 21, pp. 3801-3807, 1996.

[24] J. Artigas, A. Beltran, C. Jiménez et al., "Application of ion sensitive field effect transistor based sensors to soil analysis," Computers and Electronics in Agriculture, vol. 31, no. 3, pp. 281293, 2001.

[25] J. Artigas, C. Jiménez, C. Domínguez, S. Mínguez, A. Gonzalo, and J. Alonso, "Development of a multiparametric analyser based on ISFET sensors applied to process control in the wine industry," Sensors and Actuators B, vol. 89, no. 1-2, pp. 199-204, 2003.

[26] A. Llobera, R. Wilke, and S. Büttgenbach, "Poly(dimethylsiloxane) hollow Abbe prism with microlenses for detection based on absorption and refractive index shift," Lab on a Chip, vol. 4, no. 1, pp. 24-27, 2004.

[27] European Commission Regulation (EEC), "Community methods for the analysis of wines," Tech. Rep. 2676/90, EEC, Brussel, Belgium, 1990. 
[28] S. M. Scott, D. James, and Z. Ali, "Data analysis for electronic nose systems," Microchimica Acta, vol. 156, no. 3-4, pp. 183-207, 2006.

[29] ISO 8589, "Sensory analysis. General guidance for the design of test rooms," 2007.

[30] M. Gutiérrez, A. Llobera, A. Ipatov et al., "Application of an E-tongue to the analysis of monovarietal and blends of white wines," Sensors, vol. 11, no. 5, pp. 4840-4857, 2011.

[31] M. Gutiérrez, C. Domingo, J. Vila-Planas et al., "Hybrid electronic tongue for the characterization and quantification of grape variety in red wines," Sensors and Actuators B, vol. 156, no. 2, pp. 695-702, 2011. 

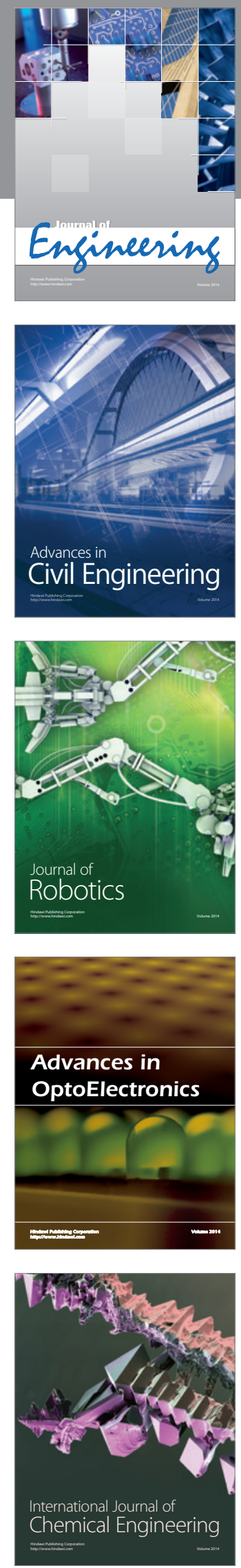

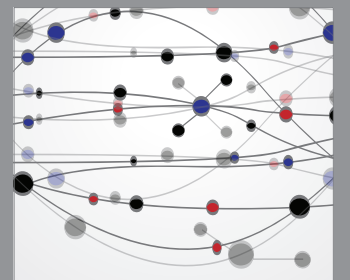

The Scientific World Journal
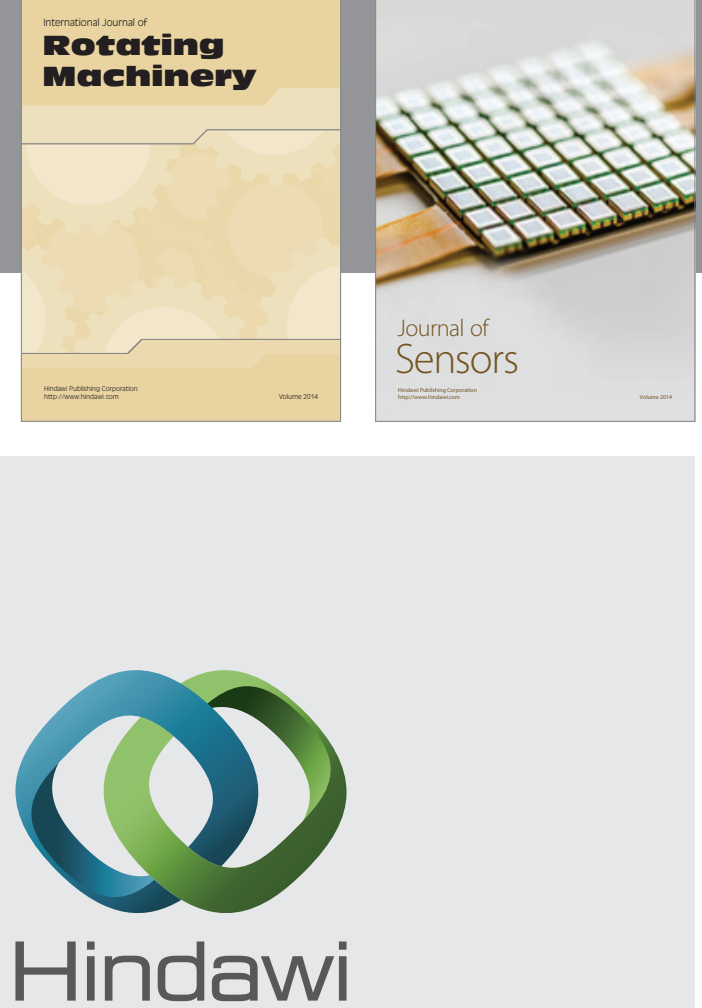

Submit your manuscripts at http://www.hindawi.com
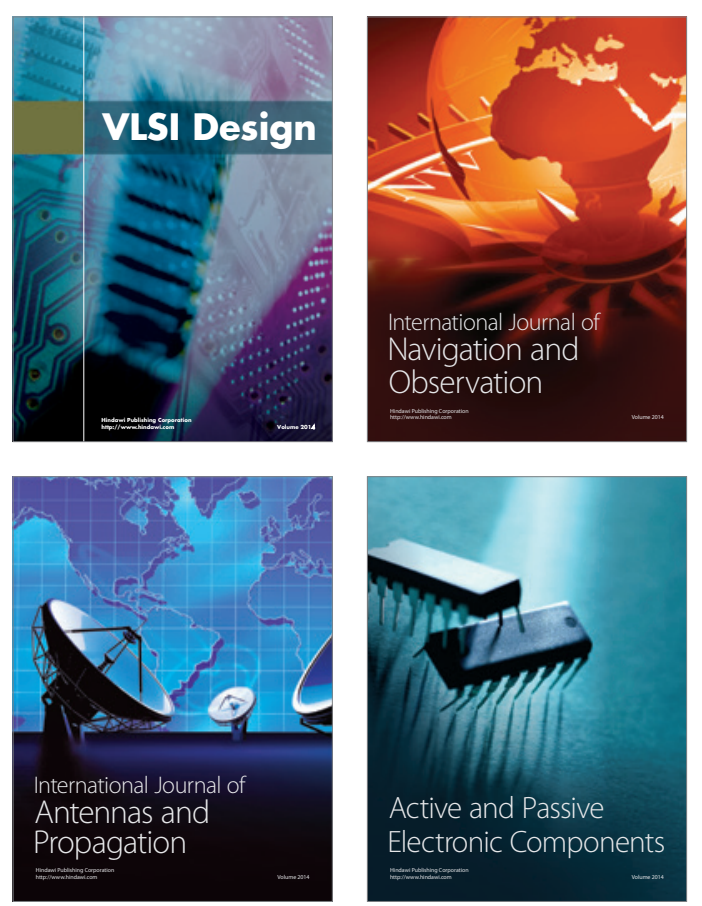
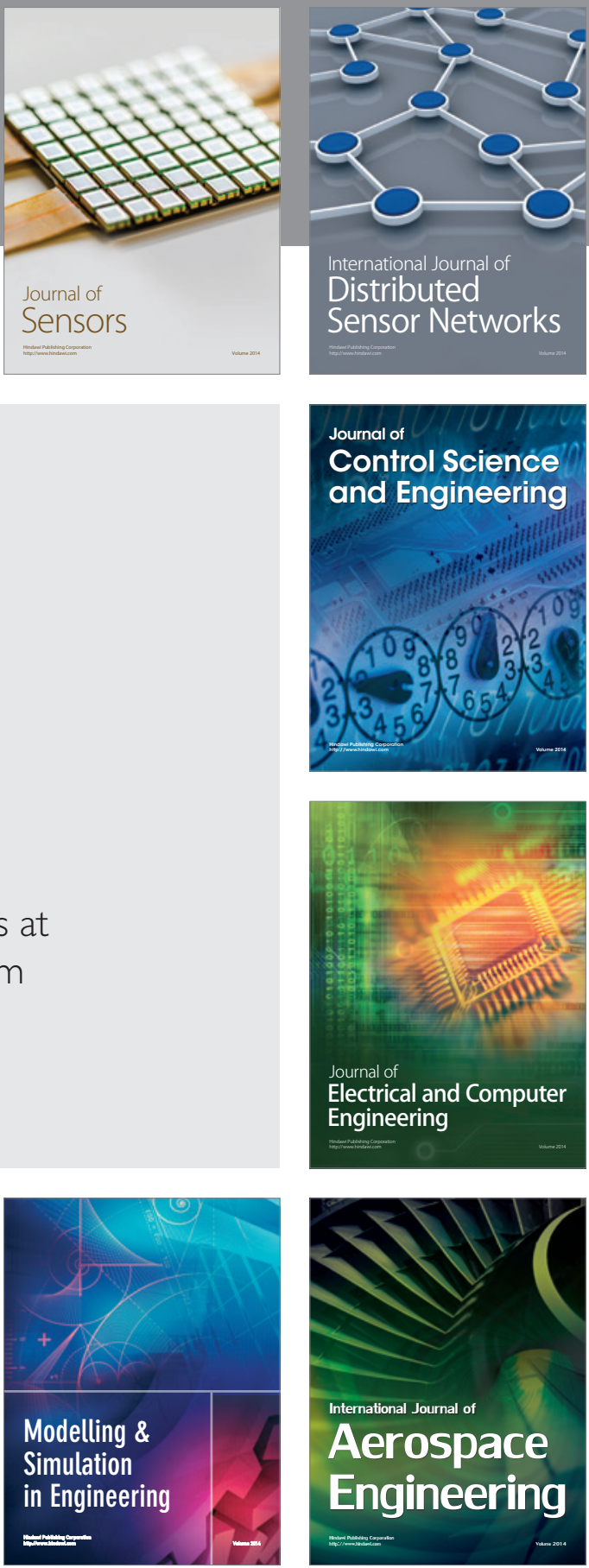

Journal of

Control Science

and Engineering
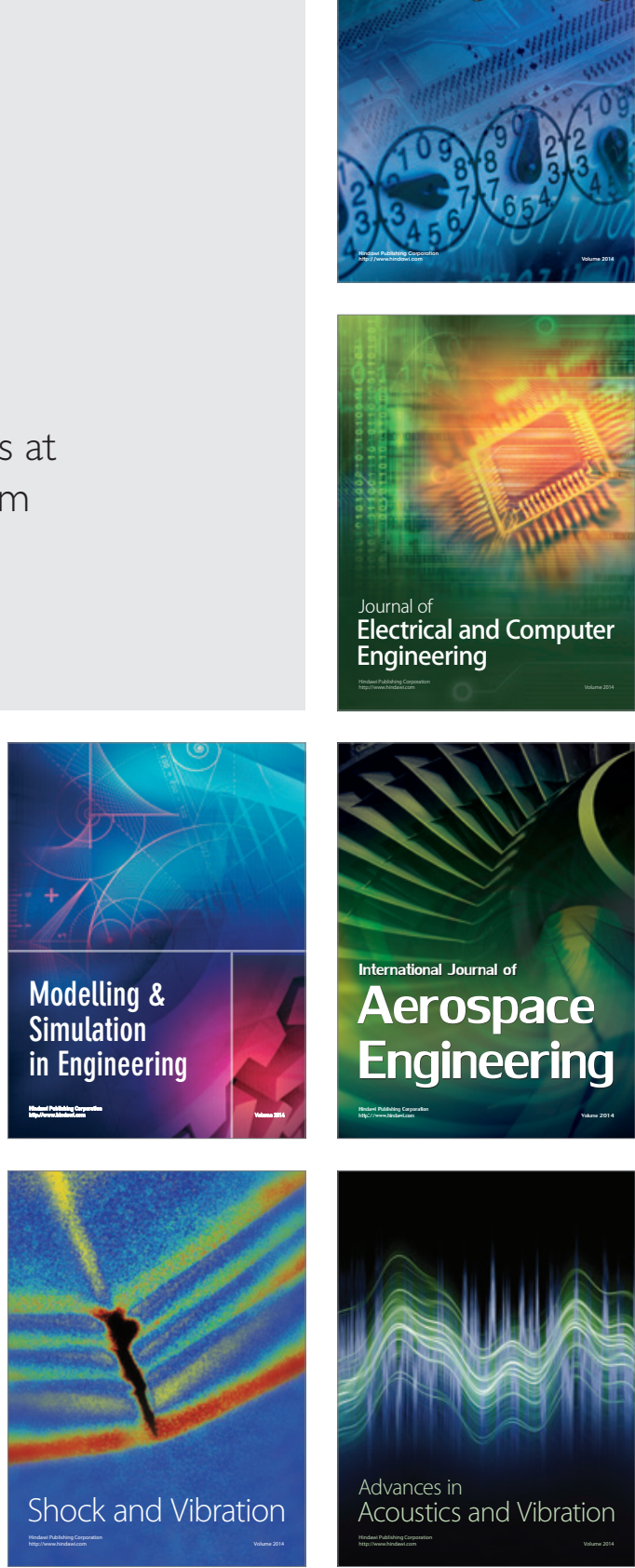